Rev. Biol. Trop., 47(4): 799-808, 1999

www.ucr.ac.cr www.ots.ac.cr www.ots.duke.edu

\title{
Criptofauna en rocas de Punta Nizuc, Caribe mexicano y su utilidad como biomonitor potencial
}

\author{
Concepción Campos-Vázquez, Luis F. Carrera-Parra, Norma Emilia González y Sergio I. \\ Salazar-Vallejo ${ }^{1}$
}

Depto. Ecología Acuática, ECOSUR, Apdo. Postal 424, Chetumal QR. 77000 México. Fax 98320447.

Recibido 18-I-1999. Corregido 7-V-1999. Aceptado 34-VI-1999.

\begin{abstract}
To complete the environmental quality assesment of Isla Mujeres-Cancún-Nizuc National Marine Park, the potential of rock cryptofauna as impact monitor was considered. A transect was defined along a visitor intensity gradient and three places (1.5-4 m deep, and 50,250 and $500 \mathrm{~m}$ from the tourist platform); in each. Inte three rocks were collected. The variations in abundance and biomass along an environmental gradient, and the relationship among diversity and dominance with the distance from the platform, density of rocks and depth were studied. Indices based on $\log 10$ (biomass +1 ), and abundance-biomass curves were used to determine environmental quality. The Bray-Curtis index and the Jaccard coefficient were used to find affinities between stations. To evaluate the functionality of "taxonomic sufficiency" in coral bottom, the Berger-Parker indices for species and families were compared. Among the 1368 organisms found, the groups with higher specific richness were polychaetes (64 spp.), mollusks (46 spp.) and crustaceans (36 spp.). We identified a highly stressed environment near to the platform (low diversity, $\mathrm{ABC}$ curve). The trend is not associated with depth (although there are increases) or rock density. Mollusk or crustacean biomass had not tendency; polychaete biomass is promissory but more evaluations are required. The cluster analysis separated rocks collected near the platform (stressed environment) from the rest. Comparison of the Berger-Parker index using species or families showed similar curves but work at family level increases differences between rocks from the same place: there is a loss of useful information when higher taxa are used.
\end{abstract}

Key words: Cryptofauna, biomonitors, Mexican Caribbean.

Desde mediados de los años 70, se empezó a cuestionar la concepción del arrecife coralino como un sistema delicado y frágil, susceptible de ser muy perjudicado casi por cualquier actividad natural o humana. En esos años, empezó a concebirse al sistema arrecifal como parte de un sistema expuesto a variada e intensa energía, motivada por la frecuencia de los huracanes y otras tormentas tropicales, que repercuten por el oleaje, incrementos en la precipitación y resuspensión y movimiento masivo de sedimentos. Así los arrecifes y otros ecosistemas tropicales son considerados como un mosaico heterogéneo, cuyas partes presentan respuestas distintas después de una perturbación mayor (Grigg y Dollar 1990, Karlson y Hurd 1993). Lo que no estaba claro era cuál podría ser el nivel de impacto, directo o indirecto, de las actividades humanas capaces de amenazar la permanencia del sistema arrecifal.

Se ha documentado con consistencia el impacto del enriquecimiento por materia orgánica. En arrecifes sujetos a incrementos en la sedimentación, se reduce el reclutamiento (Cortés y Risk 1985) y el aporte de nutrimentos ocasiona efectos variados sobre la comunidad arrecifal e incluso sobre pastos y macroalgas (Duarte 1995). Con aportes de sedimentos 
por drenaje, se incrementa la cantidad de bacterias y la producción primaria, que podrían promover un incremento en los suspensívoros. Rose y Risk (1985) asociaron un incremento en 500\% de la biomasa de la esponja perforadora Cliona delitrix con los incrementos de hasta $600 \%$ de las poblaciones de bacterias, fertilizadas por el aporte de drenaje; la bioerosión por este perforador depende del nivel de contaminación orgánica del arrecife. Esta esponja produce limo, por lo que consideraron que el impacto indirecto del drenaje modificaba el balance de carbonatos del arrecife y que este cambio se relacionaba en forma directa con el aporte de drenaje al arrecife. Rützler (1975) consideró que hasta el $98 \%$ del sedimento generado (250 g. $\mathrm{m}^{-2}$.año) por esponjas perforadoras permanecía en forma particulada en los arrecifes de plataforma. Entonces, además de la carga de sedimentos inherente al drenaje, habría otra cantidad importante de sedimentos finos producidos como resultado de la perforación del coral.

Otra consecuencia del incremento en la cantidad de alimento para los suspensívoros, es que pueden sobrecompetir al coral y dominar el espacio (Pastorok y Bilyard 1985); el resultado es que los arrecifes cambian de arrecifes coralinos a arrecifes de esponjas (Zea 1994). Cuando el aporte de drenaje es crónico puede observarse que los corales Porites astreoides, $P$. porites, Siderastrea radians y Agaricia agaricites pueden hacerse las especies de mayor abundancia y las formas resistentes a la sedimentación, como Montastrea cavernosa y Meandrina meandrites, tienden a desaparecer (Tomascik y Sander 1987).

Menos documentado pero quizá de la misma importancia, el impacto de golpeo o tocamiento por los bañistas también tiene efectos severos sobre el arrecife (Tilmant 1987). Allison (1996) encontró que el impacto físico en un mes, resultaba en fracturas en un $17 \%$ de corales susceptibles (formas ramificadas o foliosas), y en una reducción de hasta $7 \%$ de la cobertura coralina total. Otros estudios no presentan un cuadro tan impactante pero se ha indicado que el efecto de unnos 100000 visitan- tes/sitio/año, ocasionaría severos daños al arrecife (Hawkins y Roberts 1992).

En este marco de referencia, se percibe la importancia de evaluar el estado de salud del sistema arrecifal y el tratar de explicar su estado ambiental con alguno de los factores conocidos de impacto. En este trabajo presentamos un análisis de la criptofauna en rocas coralinas como mecanismo para determinar o confirmar la calidad ambiental de varios sitios a lo largo de un gradiente de perturbación, y al hacerlo evaluamos su potencial como herramienta biomonitora.

La criptofauna puede usarse como monitor de la calidad ambiental, en particular de la intensidad de la sedimentación o de la resuspensión del sedimento, porque el incremento de las partículas en la columna de agua puede ocasionar cambios en la composición trófica de los grupos presentes. Así, se considera que en condiciones de poca perturbación, hay una cierta proporción de suspensívoros y sedimentívoros y que cuando se incrementa el aporte o la resuspensión de sedimentos, se esperarían cambios en la proporción de los grupos tróficos mencionados. Así, es posible que el incremento de las partículas promueva la proliferación de algunos grupos tolerantes o que reduzca la abundancia de los grupos sensibles. Por ello, es interesante evaluar los posibles cambios en la composición trófica a lo largo de un gradiente ambiental.

\section{MATERIALES Y MÉTODOS}

En Punta Nizuc $\left(21^{\circ} 02^{\prime} \mathrm{N}, 86^{\circ} 44^{\prime} \mathrm{W}\right)$, Caribe mexicano, consideramos un transecto a lo largo del gradiente natural potencial producido por la corriente costera que se mueve al sur, de acuerdo con los alisios. Se seleccionaron tres sitios con distinta intensidad de visitantes y los clasificamos en relación con la distancia a la plataforma turística, como de alto impacto ( $50 \mathrm{~m}$ al $\mathrm{N}$ de la plataforma), de impacto moderado ( $250 \mathrm{~m}$ al $\mathrm{S}$ de la plataforma), y de impacto reducido o imperceptible $(500 \mathrm{~m}$ al $\mathrm{S}$ de la plataforma). En cada uno de los lu- 
gares determinamos la profundidad, el tipo de paisaje submarino, y el espesor del sedimento en las inmediaciones del sitio en donde se recogieron las piedras. Se recolectaron 3 rocas coralinas para examinar su criptofauna considerando que serían réplicas en el mismo lugar; en las inmediaciones de cada roca, recogimos muestras de sedimento para determinar la cantidad de materia orgánica presente. Cada roca fue recogida con una bolsa de plástico con asas, de malla fina ( $<0.5 \mathrm{~mm}$ abertura), tratando de recoger también todos los organismos refugiados debajo de su superficie.

En la playa, cada roca fue fotografiada, medida con cinta métrica en sus ejes principales y se caracterizó su tipo como coral o laja. Se determinó su volumen por desplazamiento en un recipiente graduado $(0.251 \mathrm{de}$ precisión), así como su peso y densidad para posteriormente fragmentarla. Todos los organismos encontrados fueron anestesiados con choque osmótico (5-10 min en agua de la llave) y fijados con formalina comercial al $10 \%$ en agua de mar.

En el laboratorio, las muestras se lavaron con agua de la llave por $24 \mathrm{~h}$ para quitar el exceso de formalina y de agua de mar. Luego se pusieron en alcohol etílico al $70 \%$ en agua destilada para separar grandes grupos. Con la literatura adecuada, cada grupo fue identificado hasta especie; todas las especies se depositaron en la colección de referencia de ECOSUR (QNR.IN.021.0497). Los organismos se contaron y pesaron (peso húmedo) en una balanza analítica (Sauter, 0.0001 g precisión). El contenido de materia orgánica del sedimento fue determinado por el método de calcinación de Dean (1974), para cada una de las réplicas y luego se hizo un promedio de los valores de cada sitio.

Se obtuvieron los valores de abundancia y biomasa por especie por roca con los cuales se procedió a realizar los análisis estadísticos. Se probaron algunos métodos cuantitativos recomendados para determinar la calidad ambiental en fondos blandos (de aguas templadas) que en orden decreciente son: la biomasa de los moluscos*, el número de especies, la abundancia*, la biomasa de poliquetos*, la biomasa total*, la biomasa de crustáceos* $\left(* \log _{10}\right.$ variable +1$)$. Además se realizaron análisis de curvas ABC (Warwick 1986). Estos métodos se basan en dos generalizaciones: a) la dominancia se reduce al aumentar la diversidad (ver Birch 1981 para una crítica interesante), y b) las especies corpulentas son menos abundantes que las especies de cuerpo reducido y aunque quizá el uso energético de cada población sea independiente del tamaño corporal, puede depender de su abundancia (Cotgreave 1993). Algunos estudios clásicos en fondos blandos indican que la talla corporal tiende a ser mayor (pero menor la densidad) en los sitios de menor impacto por materia orgánica y por ello pueden usarse las proporciones entre la biomasa y la abundancia como indicadoras de la calidad ambiental (Salazar-Vallejo 1991). Sin embargo, algunos estudios finos no confirman estos hallazgos; en efecto, Weston (1990) consideró que las tendencias aparentes en la variación en el tamaño corporal podrían atribuirse a deficiencias en el muestreo para recoger organismos raros y que, contrariamente a lo esperado, entre los miembros de la misma especie, los organismos de mayor tamaño se presentaban en los sitos más enriquecidos, pero se confirmó que los miembros de distintas especies tienden a ser mayores en sitios con escaso o nulo enriquecimiento.

Se realizaron análisis de regresiones para comparar la posible relación entre los datos analizados y las diferentes variables analizadas (Statistica, ver. 4.3). También se analizaron algunos descriptivos de la comunidad como lo son los índices de diversidad de Margalef, Shannon-Wiener, dominancia de Berger-Parker; y para determinar las afinidades entre sitios se computaron los índices de Jaccard y de Bray-Curtis con el programa ANACOM (de la Cruz-Agüero 1994). También se prepararon, para comparación gráfica, los resultados que se obtienen cuando el análisis se basa en las familias y al usar las especies, para evaluar la funcionalidad de la suficiencia taxonómica (Ferraro y Cole 1990) en ambientes coralinos. 


\section{RESULTADOS}

\section{Caracterización de los sitios de muestreo:} SITIO I.- Plataforma Nizuc.

Es una zona somera, de $1.5-2 \mathrm{~m}$ de profundidad y se encuentra a unos $30 \mathrm{~m}$ de la playa y a $50 \mathrm{~m}$ al Norte de la plataforma usada por los turistas, presenta numerosos parches coralinos que forman parte de un pequeño arrecife "barrera". Esta área tiene una carga de visitantes de 3000-4000/día en temporada alta y de 2000-3000 en temporada baja. Hay muchos corales muertos como Montastraea annularis y Diploria sp, además de la proliferación de algas filamentosas y cianobacterias que crecen sobre las rocas coralinas y sobre las macroalgas; sobresale la feofita Dictyota. Entre los parches coralinos se localizan lechos arenosos y de pastos marinos (Thalassia testudinum), dispersos en ellos se encuentran individuos de la clorofita Penicillus. Sobre el lecho rocoso, hay una capa de sedimento de un espesor promedio de $2.3 \mathrm{~cm}$. En este lugar se recolectaron las rocas uno, dos y tres.

SITIO II.- A $250 \mathrm{~m}$ de distancia de plataforma Nizuc.

Es un área somera, de 2-2.5 m de profundidad, cercana al canal de navegación, o ruta que siguen las embarcaciones al salir de la laguna Nichupté. Se localiza a unos $250 \mathrm{~m}$ al Sur de la plataforma turística; el fondo es rocoso, sobre el que se encuentra una capa de sedimento con un espesor promedio de $1.6 \mathrm{~cm}$. Como en el sitio anterior, el paisaje es de varios parches coralinos de distintos tamaños, pero los corales están en mejores condiciones; la vegetación predominante alrededor de ellos es Thalassia testudinum, Syringodium filiforme, Dictyota sp., Rhipocephalus sp. y Penicillus sp. siendo los pastos los dominantes.

A pesar de no ser un sitio muy visitado por turistas, se observó que algunos parches arrecifa- les, a poca profundidad, tenían daños severos por choque de propelas; de la misma forma se pudieron apreciar algunos corales como Siderastrea siderea parcialmente blanqueados. En este sitio se tomaron las rocas cuatro, cinco y seis.

SITIO III.- A $500 \mathrm{~m}$ de distancia de plataforma Nizuc.

Es un área con mayor profundidad (4 m) que está a $500 \mathrm{~m}$ en dirección sur de la plataforma turística y a $1 \mathrm{Km}$ de la principal estructura arrecifal de tipo barrera, localizada en dirección este. El lecho rocoso estaba cubierto con sedimento de un espesor de $1 \mathrm{~cm}$. Los parches coralinos presentes aquí básicamente eran monoespecíficos del coral de ciervo (Acropora cervicornis) y mostraba blanqueamiento parcial en menos del $20 \%$ de las colonias; otras especies presentes (Siderastrea radians y Porites astreoides) denotaron signos de blanqueamiento parcial o total. La vegetación dominante fue Thalassia testudinum bastante dispersa y de poca altura y Penicillus sp. Por las condiciones observadas se considera que el sitio no experimenta un impacto humano directo e importante; sin embargo, por la pedacería de corales cuerno de ciervo observada, es probable que las tormentas sean responsables de esa perturbación. Se recolectaron en este sitio las rocas siete, ocho y nueve.

Análisis faunístico: La fauna críptica del total de las rocas procesadas incluye 218 especies (el listado de las especies y los datos de cada roca estan disponible con los autores). Los 1368 organismos identificados se reparten como se muestra en el cuadro 1 .

\section{DISCUSIÓN}

Para la realización de los análisis estadísticos de las comunidades, solo se tomaron en cuenta los organismos que se encontraron con

\section{CUADRO 1}

Número de especies de los principales grupos taxonómicos de la criptofauna de rocas de Punta Nizuc.

$\begin{array}{ccccccccc}\text { Esponjas } & \text { Antozoos } & \text { Moluscos } & \text { Poliquetos } & \text { Crustáceos } & \text { Equinodermos } & \text { Sipunculos } & \text { Ascidias } & \text { Peces } \\ 6 & 6 & 46 & 64 & 36 & 24 & 28 & 9 & 2\end{array}$




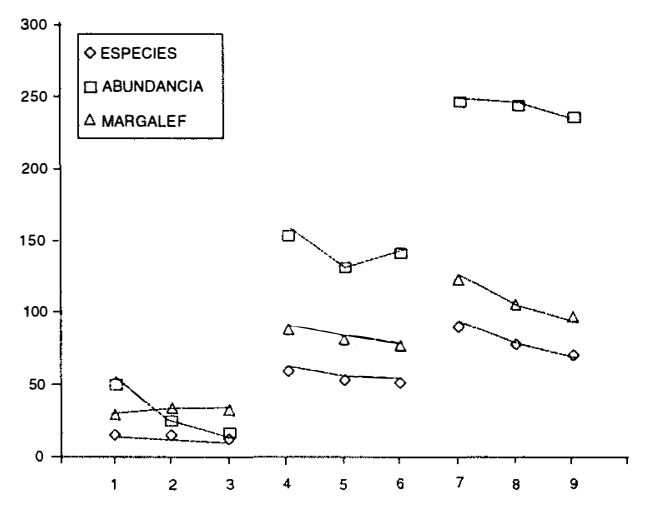

Fig. 1. Relación de la riqueza de especies, abundancia e índice de diversidad de Margalef por roca muestreada.

vida, eliminado de dichos análisis las especies de moluscos que se registraron por la presencia sólo de sus conchas.

Los índices de Margalef, riqueza específica y abundancia, mostraron una marcada tendencia hacia el incremento en relación directa con la distancia de la plataforma, presentandose los valores más bajos en el sitio cercano a la misma, o sea el de mayor impacto por turismo y los valores más altos en los sitios con menor y nulo impacto antrópico (Fig. 1). Mediante un análisis de regresión se puede apreciar una relación directamente proporcional entre la diversidad de Shannon-Wiener y la distancia a la plataforma turística y una relación inversa entre el índice de Berger-Parker con la distancia a la plataforma, reforzando la idea de que la primera zona presenta perturbación (Fig. 2). Warme (1975) estudió la criptofauna y sus variaciones a lo largo del arrecife encontrando que los sitios más protegidos tenían una fauna más rica que sitios semejantes en el arrecife abierto o en el arrecife frontal, caso contrario a lo presentado en este estudio, lo cual se puede explicar por la gran presión antrópica que existe en la zona más protegida.

La biomasa total de los organismos presentó un incremento en relación con la distancia; los valores máximos están en la zona de menor influencia de turismo. En este caso, a diferencia de los fondos blandos, los moluscos no podrían recomendarse como monitores por

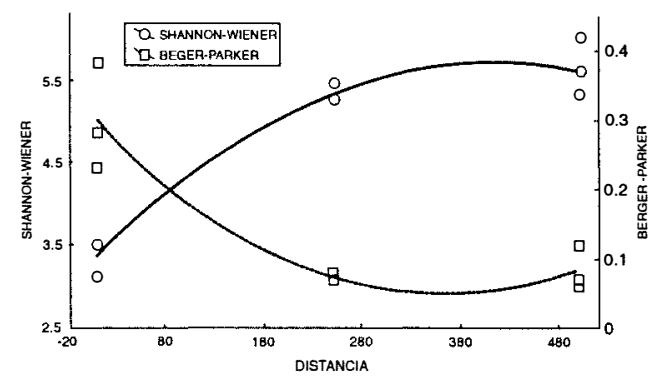

Fig. 2. Relación entre el índice de diversidad de ShannonWiener y de dominancia de Berger- Parker contra el gradiente de distancia a la plataforma.

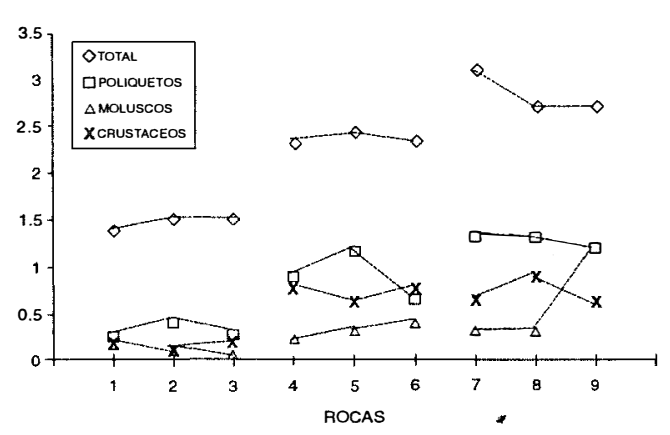

Fig. 3. Relación de la biomasa $(\log 10$ biomasa +1$)$ por roca muestreada.

la poca diferencia que se presenta entre los sitios; los crustáceos podrían ser una mejor opción, pero además de no presentar una tendencia clara, en su mayoría son especies oportunistas para la colonización de las rocas más que perforadores de las mismas; el único grupo que presentó resultados que podrían explicar las diferencias entre los sitios fue el de los poliquetos, su biomasa mostró tendencias similares a las indicadas por los índices de diversidad, resaltando las marcadas diferencias entre los diferentes sitios muestreados (Fig. 3).

$\mathrm{El}$ análisis de las curvas $\mathrm{ABC}$ mostró que el área cercana a la plataforma turística presenta un estado de perturbación considerable; la curva de la abundancia estuvo por encima de la biomasa; todo lo contrario en los dos restantes sitios los cuales se puede considerar como sitios no perturbados (Fig. 4)

Para comprobar que los valores analizados no estaban siendo influenciados por variables distintas al gradiente de perturbación por los 

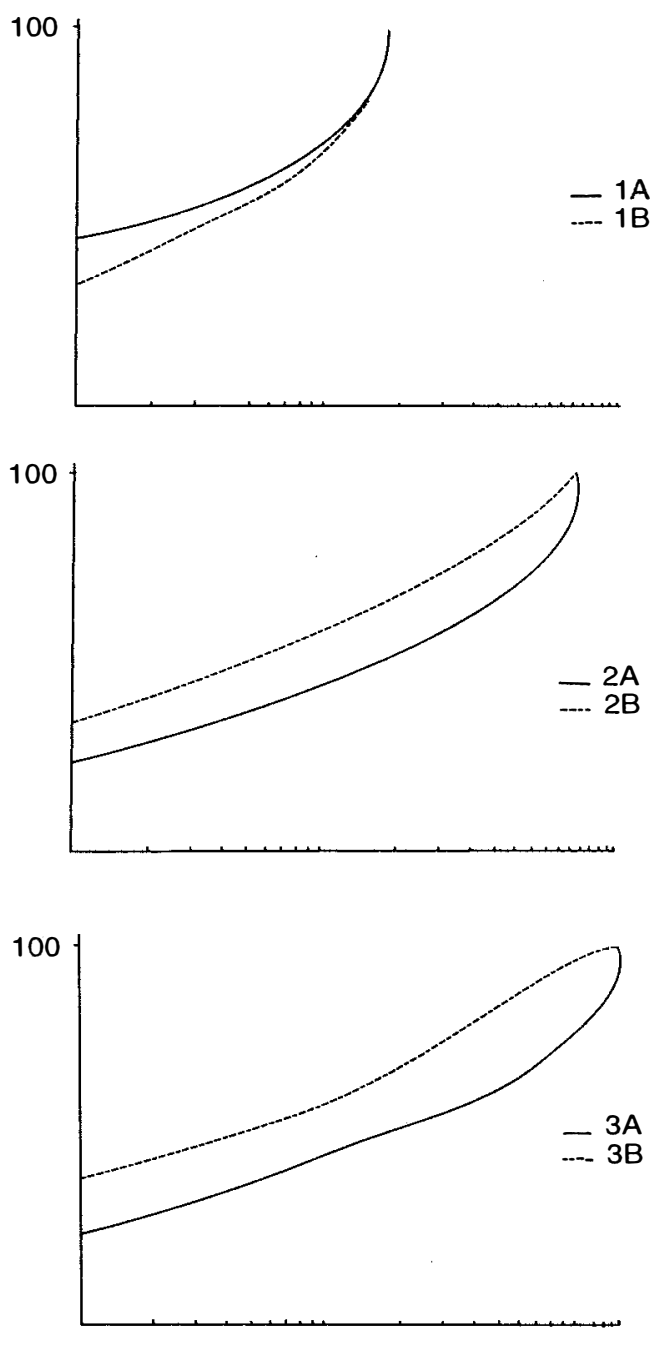

RANGO

Fig. 4. Análisis de las curvas ABC. El sitio cercano a la plataforma turística presenta perturbación considerable, los dos restantes son considerados como sitios no pertur-

turistas entre el primero y el último sitio, se realizaron análisis de regresión tratando de explicar las posibles variaciones con respecto a estas variables. En la comparación del efecto de la profundidad entre los sitios muestreados, los resultados indican un incremento directo entre esta y la diversidad (e inverso entre la dominancia y la profundidad) (Fig. 5); sin embargo, las diferen-

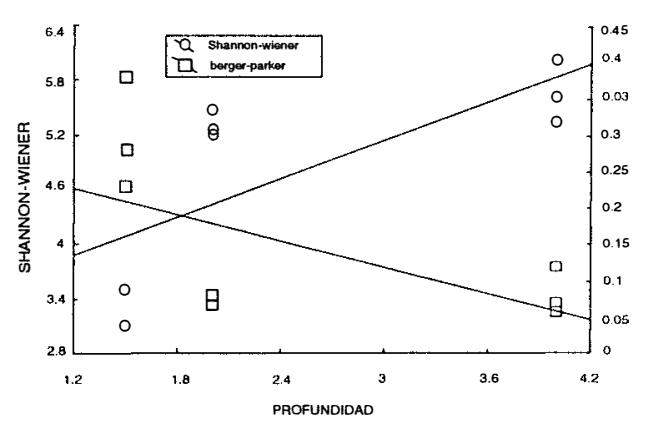

Fig. 5. Relación entre el índice de diversidad de ShannonWiener y de dominancia de Berger-Parker contra la profundidad.

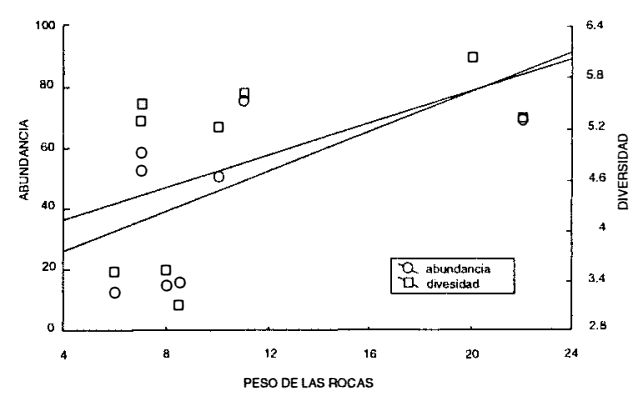

Fig. 6. Relación entre la abundancia y la diversidad contra el peso de las rocas.

cias entre los sitios I y II son muy marcadas, a pesar de tener casi la misma profundidad. Por lo tanto, más que el efecto de la profundidad, la baja diversidad (y alta dominancia) en la zona cercana a la plataforma se explica por la influencia de factores antrópicos directos (resuspensión, golpeteo, etc.). Esta misma tendencia se observó en relación con el peso de las rocas con la diversidad y la abundancia, a pesar de existir una relación directa entre estas; rocas de pesos similares (7-8 kg) de los sitios I y II presentaron una gran diferencia en los valores de diversidad y abundancia (Fig. 6). Otra posibilidad sería el efecto de estancamiento del agua, con posibles problemas de calentamiento y falta de oxígeno, pero como no hubo diferencias en la cantidad de materia orgánica, la desechamos. Es deseable contar con mayor información de las variaciones en nutrimentos e influencia fecal en la zona para determinar con mayor detalle las razones de esta baja diversidad; la proximidad a la playa obliga esta consideración. 


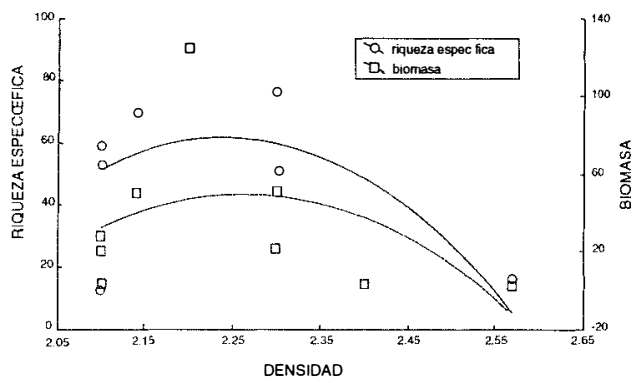

Fig. 7. Relación entre la riqueza de especies y la biomasa $(\log 10$ biomasa +1$)$ contra la densidad de las rocas.

La densidad de las rocas es otra variable que podría influir en los resultados obtenidos, ya que una roca menos densa podría ser perforada con más facilidad. Se realizó un análisis de regresión entre la riqueza específica y la biomasa contra la densidad de las rocas; el resultado fue una relación de tipo exponencial negativa que no refleja las diferencias entre los sitios, lo cual nos permite afirmar que la densidad de las rocas no es un factor determinante de la composición de la criptofauna (Fig. 7).

Para evitar la posible confusión en la interpretación del análisis previo, ya que se agrupan organismos perforadores con oportunistas que no necesariamente reflejan la posible resistencia de la roca, y ya que no todas las especies encontradas son realmente perforadoras, se realizaron otros análisis considerando sólo la abundancia y biomasa de perforadores $\left(\log _{10}\right.$ biomasa +1$)$. Se eligieron a los sipuncúlidos y a los poliquetos sabélidos y se relacionaron con la densidad de las rocas y con la distancia a la plataforma. Los resultados de la relación con la densidad no muestran tendencia alguna; por ello, confirmamos que la densidad de las rocas no explica los cambios observados. Sin embargo, el gradiente de la distancia, que refleja un supuesto gradiente de impacto antrópico, si explica los cambios presentes (Figs. 8,9). Hutchings (1986) afirmó que la densidad de las rocas modifica las tasas de bioerosión y consideró que las de mayor densidad tienden a ser más perforadas; es posible que haya querido decir que dichas rocas pierden más peso por unidad de tiempo, con lo que no habría diferencia importante entre los perforadores de rocas de distinta densidad.
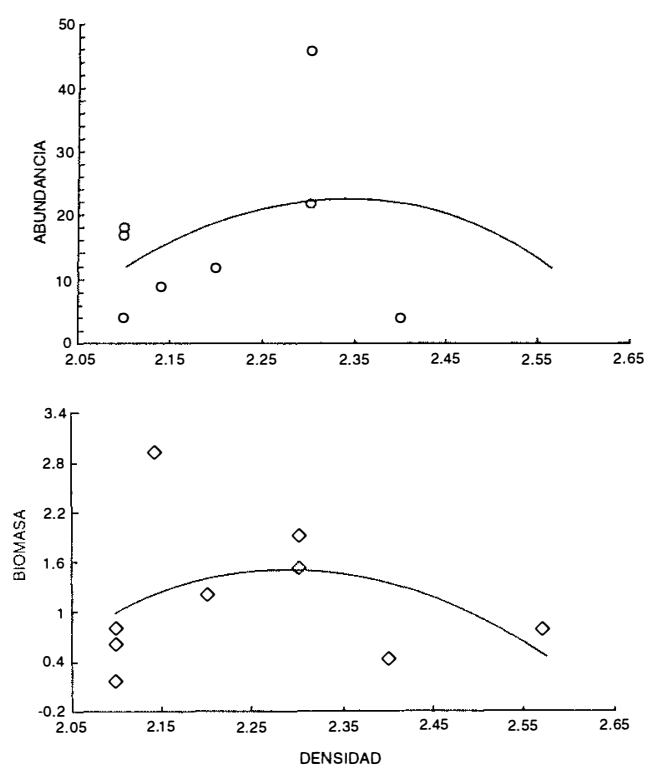

Fig. 8. Relación entre la abundancia y la biomasa $(\log 10$ biomasa +1 ) de organismos perforadores (sipuncúlidos y poliquetos sabélidos) contra la densidad de las rocas.
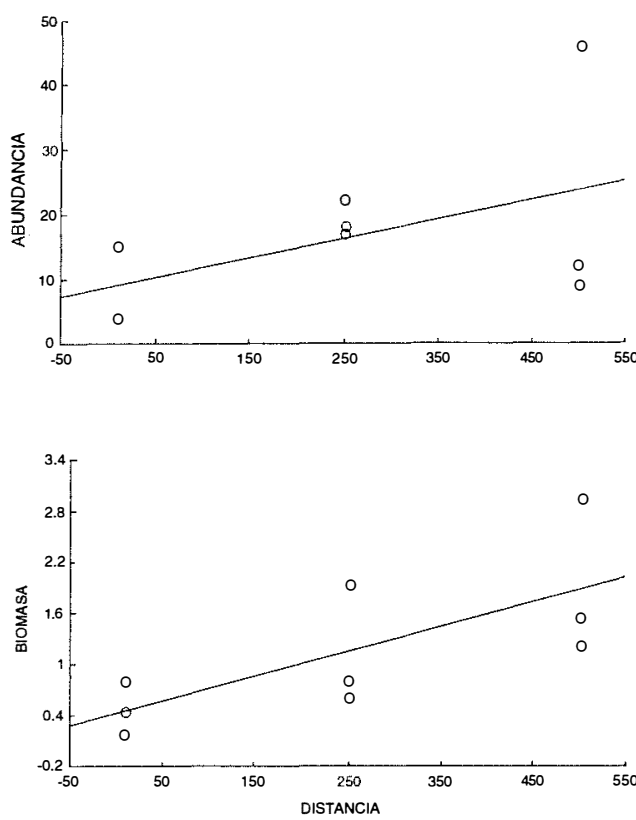

Fig. 9. Relación entre la abundancia y la biomasa $(\log 10$ biomasa +1 ) de organismos perforadores (sipuncúlidos y poliquetos sabélidos) contra el gradiente de distancia a la plataforma. 

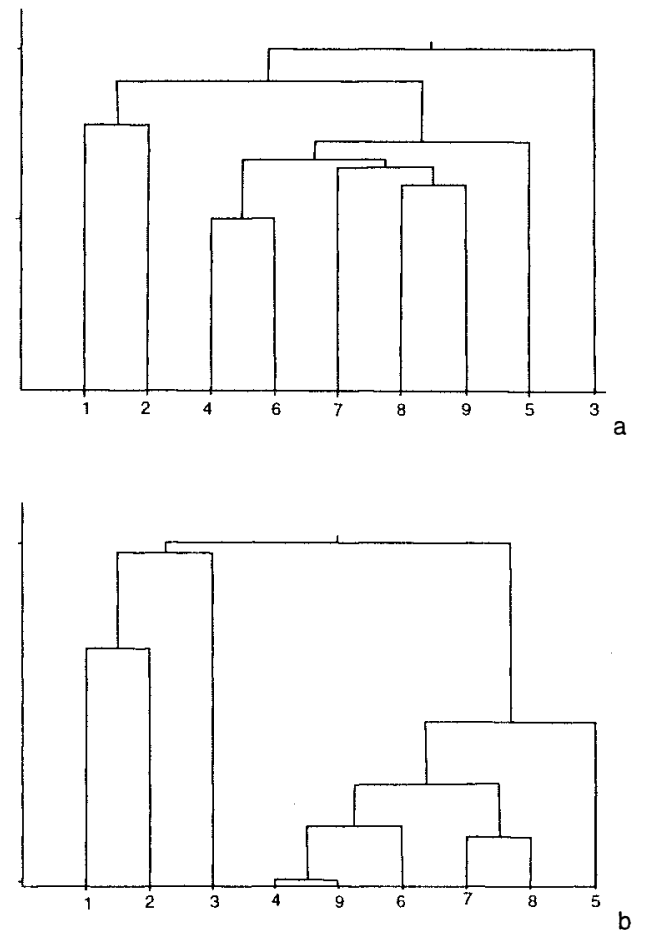

Fig. 10. Dendrogramas: a) Indice de disimilitud de BrayCurtis, b) Indice de Jaccard.

Con el análisis de agrupamiento por abundancias se reconocen dos grandes grupos, el primero, con un grado de disimilaridad menor, fue formado por las rocas recolectadas en los sitios II y III (rocas 4-9, zonas de mediano y nulo impacto antrópico), quedando aisladas las tres rocas recolectadas en las cercanías de la plataforma (Fig. 10a). Además, se realizó el análisis de similitud de presencia-ausencia con el índice de Jaccard y se confirmó el resultado; las rocas del sitio I (rocas 1-3; intenso impacto antrópico) quedan aisladas del resto (Fig 10b). Esto confirma la diferencia en la calidad ambiental, reflejada por los valores de riqueza específica y de abundancia, entre las inmediaciones de la plataforma y los sitios más alejados de la misma. Hay que mencionar que las diferencias encontradas entre las rocas de un mismo sitio no son más grandes que las encontradas entre los sitios.

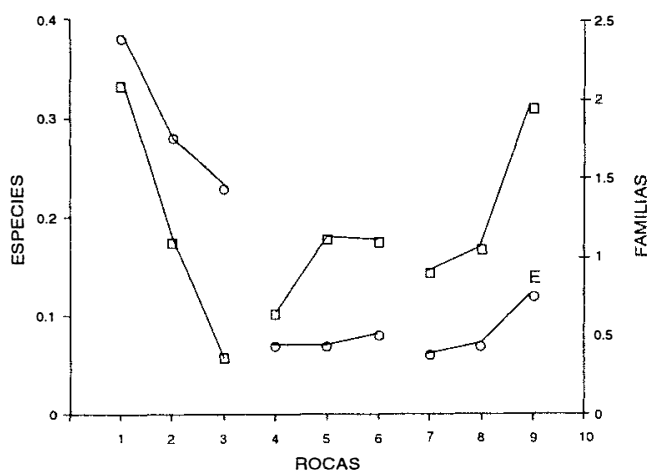

Fig. 11. Indice de dominancia de Berger-Parker por especies y familias en relación a las rocas muestreadas.

Para evaluar la funcionalidad de la suficiencia taxonómica en fondos coralinos, se compararon los resultados de dominancia de Berger-Parker usando por separado las especies y las familias (Fig. 11). El resultado muestra un comportamiento similar en la tendencia de las curvas, pero hay diferencias importantes; la curva de familias indica diferencias muy marcadas entre las rocas de un mismo sitio. Al utilizar los valores a nivel de especie, se percibe mayor semejanza entre las rocas del mismo sitio por lo que nos parece un indicador más fino de la calidad ambiental. El tratar de identificar los organismos sólo a familia, con evidentes ahorros de tiempo y dinero, puede ocasionar una importante pérdida de información sobre las pequeñas variaciones que serían exageradas con cualquier índice usado sólo la familia. En el medio tropical, donde conocemos muy poco sobre las variaciones naturales en las comunidades, el uso de esa herramienta sería una decisión desafortunada porque no alcanzaríamos un adecuado nivel de conocimiento sobre la naturaleza. La posible ventaja económica se perdería fácilmente; utilizar datos a nivel de familia podría darnos resultados enmascarados que nos llevarían a conclusiones erróneas.

\section{AGRADECIMIENTOS}

Agradecemos al personal del parque marino nacional "Costa Occidental de Isla Mujeres- 
Cancún-Nizuc" en especial a Tomás Camarena Luhrs, por su interés en la realización de este ejercicio, y a otras autoridades de la SEMARNAP por el apoyo económico para esta investigación. Reconocemos el respaldo de Manuel Jesús Santamaría, para poder visitar y explorar los sitios seleccionados. Humberto Baena tomó las fotografías en el campo. El tratamiento de los materiales fue optimizado por la colaboración de Miguel A. Ruiz, Jennifer Ruiz, María Rosa Laguna, Patricia Baquedano y Angélica Jiménez. Fotocopias de artículos fueron provistas por Verónica Rodríguez y Ricardo Martínez (San Diego Marine Biology Lab.). Apoyo con el uso de ASFA en CICH-UNAM, por Josefina Galicia, y en el Instituto de Biología, UNAM por Agustina Lara. Este trabajo se realizó parcialmente con respaldo económico del CONACYT (4120-P).

\section{RESUMEN}

Para completar el diagnóstico de la calidad ambiental en el Parque Marino Nacional Isla Mujeres-Cancún-Nizuc Caribe mexicano, se estudió la posible utilidad de la criptofauna de rocas como monitora de impacto. Se trazó un transecto a lo largo de un gradiente de intensidad de visitantes a Punta Nizuc. Se visitaron tres lugares (1.5-4 m prof.), a 50, 250 y $500 \mathrm{~m}$ de distancia de la plataforma turistica, y en cada uno de ellos se recogieron tres rocas. Los 1368 organismos encontrados fueron identificados y se distribuyen en 218 especies; los grupos de mayor riqueza específica fueron los poliquetos (64 spp), los moluscos (46 spp) y los crustáceos (36 spp). Se estudió la variación en número y en biomasa a lo largo del supuesto gradiente ambiental, y también la relación de algunos descriptores de la comunidad (diversidad, dominancia) con la distancia a la plataforma, con la densidad de la roca y con la profundidad. Se analizaron algunos índices que utilizan $\log _{10}$ (biomasa +1 ) y las curvas de abundancia-biomasa para determinar la calidad del ambiente. Se realizaron análisis de agrupamiento (Bray-Curtis y Jaccard) para determinar la afinidad entre estaciones. Para evaluar la funcionalidad de la suficiencia taxonómica en fondos coralinos, se compararon los resultados de dominancia de Berger-Parker para las especies y familias. Las agrupaciones entre las rocas ilustran dos grupos principales, uno formado por las rocas recolectadas cerca de la plataforma (zona perturbada) y la otra formada por el resto de las rocas en las áreas con mediano y nulo impacto.

\section{REFERENCIAS}

Allison, W. R. 1996. Snorkeler damage to reef corals in the Maldive Islands. Coral Reefs 15: 215-218.

Birch, D. W. 1981. Dominance in marine ecosystems. Amer. Nat. 18: 262-274.

Cortés, J. \& M. J. Risk. 1985. A reef under siltation: Cahuita, Costa Rica. Bull. Mar. Sci. 36: 331-336.

Cotgreave, P. 1993. The relationship between body size and population abundance in animals. Trends Ecol. Evol. 8: 244-248.

Cruz-Agüero, A. de la 1994. ANACOM, Sistema para el Análisis de Comunidades, Versión 3.0. Departamento de Pesquerías y Biología Marina, CICIMAR-IPN. México.

Dean, W. E. 1974. Determination of carbonate and organic matter in calcareous sediments and sedimentary rocks by loss on ignition: Comparison with other methods. J. Sedim. Petrol. 44: 249-253.

Duarte, C. M. 1995. Submerged aquatic vegetation in relation to different nutrient regimes. Ophelia 41: 87 112.

Ferraro, S. P. \& F. A. Cole. 1990. Taxonomic level and sample size sufficient for assessing pollution impacts on the Southern California Bight macrobenthos. Mar. Ecol. Progr. Ser. 67: 251-262.

Grigg, R. W. \& S. J. Dollar. 1990. Natural and anthropogenic disturbance on coral reefs, p. 439-452. In Coral Reefs. Z. Dubinsky (ed.). Ecosystems of the World, 25. Elsevier, Amsterdam.

Hawkins, J. P. \& C.M. Roberts. 1992. Can Egypt's coral reefs support ambitious plans for diving tourism? Proc. 7th Intern. Coral Reef Symp., Guam. 2: 1007 1013.

Hutchings, P. A. 1986 Biological destruction of coral reefs: A review. Coral Reefs 4: 239-252.

Karlson, R. H. \& L. E. Hurd. 1993. Disturbance, coral reef communities, and changing ecological paradigms. Coral Reefs 12: 117-125.

Pastorok, R. A. \& G. R. Bilyard. 1985. Effects of sewage pollution on coral reef communities. Mar. Ecol. Progr. Ser. 21: 175-189.

Rose, C. S. \& M. J. Risk. 1985. Increase in Cliona delitrix infestation of Montastrea cavernosa heads on an or- 
ganically polluted portion of the Grand Cayman fringing reef. PSZN I, Mar. Ecol. 6: 345-363.

Rützler, K. 1975. The role of burrowing sponges in bioerosion. Oecologia 19: 203-216.

Salazar-Vallejo, S. I. 1991. Contaminación marina: Métodos de evaluación biológica. CIQRO y Gob. Quintana Roo, Chetumal, 193 p.

Tilmant, J. T. 1987. Impacts of recreational activities on coral reefs, p. 195-214. In Human Impacts on Coral Reefs: Facts and Recommendations. B. Salvat (ed.). Antenne Mus. EPHE, Polinesia Francesa.

Tomascik, T. \& F. Sander. 1987. Effects of eutrophication on reef-building corals, 2. Structure of scleractinian coral communities on fringing reefs, Barbados, West Indies. Mar. Biol. 14: 53-75.
Warme, J. E. 1975. Borings as trace fossils, and the processes of marine bioerosion, p. 181-229. In The study of trace fossils. R.W. Frey (ed.). Springer, Berlín.

Warwick, R. M. 1986. A new method for detecting pollution effects on marine macrobenthic communities. Mar. Biol. 92: 557-562.

Weston, D. P. 1990. Quantitative examination of macrobenthic community changes along an organic enrichment gradient. Mar. Ecol. Progr. Ser. 51: 233244.

Zea, S. 1994. Patterns of coral and sponge abundance in stressed coral reefs at Santa Marta, Colombian Caribbean, p. 257-264. In Sponges in time and space: Biology, Chemistry, Paleontology. R.W.M. van Soest, T.M.G. van Kempen y J.-C. Braekman (eds.). Balkema, Rotterdam. 